\title{
SCALING IN 2-D DISTRIBUTION OF TOPOLOGICAL DEFECTS IN A LIQUID CRYSTAL
}

\author{
M. MassalsKa-Arodź \\ H. Niewodniczański Institute of Nuclear Physics \\ Radzikowskiego 152, 31-342 Kraków, Poland
}

(Received June 23; 1998)

\begin{abstract}
Distribution of the topological point defects observed microscopically by Nagaya, Hotta, Orihira and Ishibashi in Schlieren texture of $N$-(4-n-methoxy benzylidene) $4^{\prime}-n^{\prime}$-butylaniline has been analyzed. The same fractal dimensionality $D_{\mathfrak{i}}=1.4$ has been estimated for several sets of defects obtained in subsequent stages of evolution of the nematic sample after the transition from the isotropic liquid phase.
\end{abstract}

PACS numbers: $61.30 . J f, 61.43 . \mathrm{Hv}, 64.70 . \mathrm{Md}$

\section{Introduction}

Apart from the case of monocrystals in condensed matter physics one deals with systems characterized by a larger or smaller degree of disorder. It turns out that in many materials the lack of translational symmetry, typical of ideal crystals, does not mean the total lack of symmetry. In spite of seemingly complete disorder a new kind of symmetry called the self-similarity can be found there [1]. Perfect self-similarity, i.e., the scale invariance, is the main geometrical feature of the deterministic fractals. Due to the hierarchical form of structure composed of various sized elements, the fractals have no characteristic length scale. For real fractal-like disordered systems the feature of scale invariance can be identified only in the statistical sense and the range of scale changes is rather limited. Nevertheless, the self-similarity of such real systems can also be quantitatively expressed by the power-law dependence between the mass $N$ (or the number of structural elements) and the linear size $L$ of the object

$$
N(L) \sim L^{D_{i}} \text {. }
$$

The $D_{\mathfrak{f}}$ exponent called fractal dimensionality (or Hausdorff-Besicovitch dimensionality) [1] is a non-integer quantity usually and replaces the integer dimensionality $d$ of the Euclidean space. The fractal distribution of structural elements in 
$d$ dimensional space can be also found by checking the dependence of density of structural elements on scale $L: \rho(L)=N /\left(L^{d}\right) \sim L^{D_{1}-d}$ detected if $D_{\mathrm{f}}$ differs from $d$.

Liquid crystalline phases possessing features of the isotropic liquid (macroscopic fluidity) as well as of the regular crystal (anisotropy of structural and physical properties) are the special examples of disordered materials. During cooling the complete rotational symmetry of an isotropic phase of elongated molecules is broken, leading to a cylindrical symmetry of uniaxial nematic phase. The transition is accompanied by the appearance of topological defects in those places of the sample where the alignment of the molecules, i.e., a versor called director reveals discontinuity [2]. In the nematic phase defects form lines called disclinations (strings or vortices), points called monopoles and textures being special arrangements of linear defects but there are techniques allowing to have only one type of defects in experiment: Due to birefringence of liquid crystalline phases the topological defects can be directly observed by the polarizing microscope. The defects in form of disclination lines running perpendicular to the plane of image are unstable. Thus, they transform into real point defects through the mechanism of "escape to the third dimension" [3]. In Schlieren texture the defects of \pm 1 type are identified as points from which four dark "brushes" emanate which is presented schematically in Fig. 1. In the areas of the sample which are seen as dark, the elongated molecules are oriented perpendicular to polarizer or analyzer. This means the di-

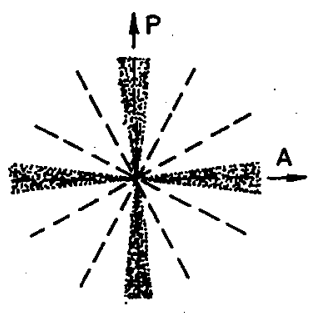

$m=1$
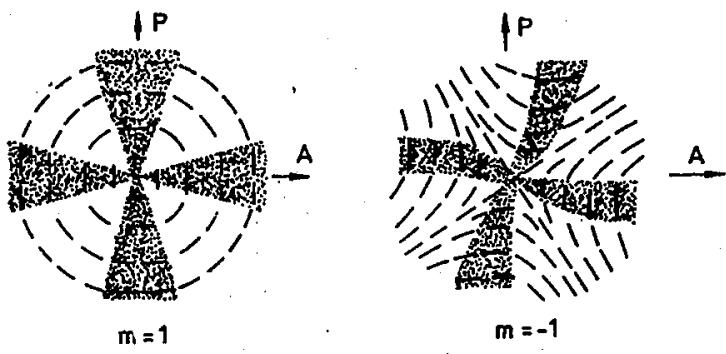

Fig. 1. Schematic presentation of topological defects of $m= \pm 1$ type appearing in Schlieren texture of liquid crystal sample. Sectors represent elongated molecules.

rector performs $2 \pi$ angle during its full turn around the disclination. Recently the coarsening dynamics of the ensemble of disclinations in nematic phases $[4,5]$ and the behavior of single defect [6] has been thoroughly studied. The aim of this paper is to check if the arrangement of the topological defects in the nematic phase reveals any fractal-like features.

\section{Results and discussion}

Nagaya, Hotta, Orihira and Ishibashi [7] haye studied the time evolution of ensemble of topological defects of \pm 1 type (obtained by the technique described in Ref. [8]) appearing in the form of Schlieren texture in transition from an isotropic to a nematic phase of $N_{-}$-(4-n-methoxy benzylidene) $4^{\prime}-n^{\prime}$-butylaniline (MBBA). 
Each dot in images of Fig. 2 represents a single point defect observed microscopically in the plane of the sample. The images have been taken in different stages of new nematic phase, i.e., in $40 \mathrm{~s}, 100 \mathrm{~s}$, and $200 \mathrm{~s}$ after the phase transition from an isotropic to anisotropic liquid phase. Each of the images illustrates the same approximately $(1 \times 1) \mathrm{mm}^{2}$ area of the sample. As the defects of the opposite sign annihilate, the number $N$ of the defects in the subsequent images is smaller and smaller. The observed relation $N(t) \sim t^{-1}$ proceeds from the fact that the magnitudes of the elastic force and the viscous force between two defects of the opposite sign are equal $[4,5,7]$.
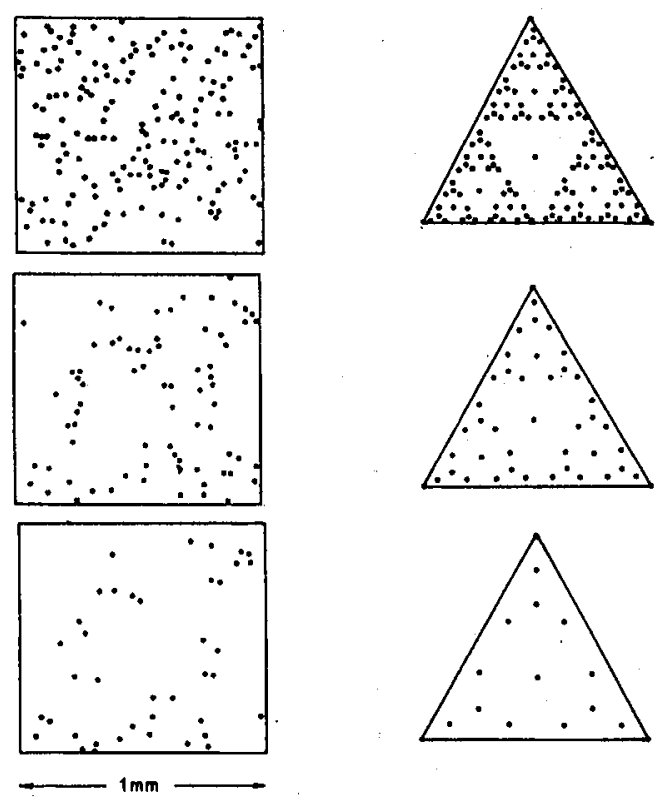

Fig. 2. Left: ensemble of dots representing the disclination lines crossing the plane of $(1 \times 1) \mathrm{mm}^{2}$ of the sample of MBBA observed in the nematic phase by means of polarizing microscope. Subsequent images present the 2 -d distribution of point defects (dots) in different stages of evolution, respectively in $40 \mathrm{~s}, 100 \mathrm{~s}$, and $200 \mathrm{~s}$ after the phase transition (after [7]). Right: the construction of the Sierpinski gasket regularity in subsequent stages of iteration characterized by different length scales is presented for illustration of the analogy with self-similar distribution of defects. Dots represent the centers of white various-sized triangles in the original black-white Sierpinski gasket.

The fact that the topological defects observed by Nagaya et al. [7] form an ensemble of points in the plane of the sample allows one to formulate the problem of quantitative description of 2-d distribution of topological defects in various stages of their time evolution after the phase transition. It seems interesting to check whether the distribution of the defects, which looks very much like random, has 
any invisible regularities. Thus, the distribution of the strength \pm 1 topological defects presented in Fig. 2 in the form of dots has been analyzed in order to check if it could be quantitatively characterized by the fractal dimensionality. For that purpose the images have been magnified to the area of $(10 \times 10) \mathrm{cm}^{2}$. Next, several squares of side $L$ ranging from $1 \mathrm{~cm}$ to $10 \mathrm{~cm}$ have been prepared. The maximum number $N$ of points found in the square of side $L$ has been counted for all squares. Such procedure has been repeated for subsequent images taken in subsequent moments of evolution of the defect pattern. In Fig. 3 the dependence of $N(L)$ for several stages of evolution of the nematic phase of MBBA is presented in the double logarithmic coordinates. Additionally, the density $\log N /(L \times L)$ vs. $\log L$ is shown for the ensemble of defects in two extremal stages analyzed, i.e., at $40 \mathrm{~s}$ and at $200 \mathrm{~s}$ after the phase transition.

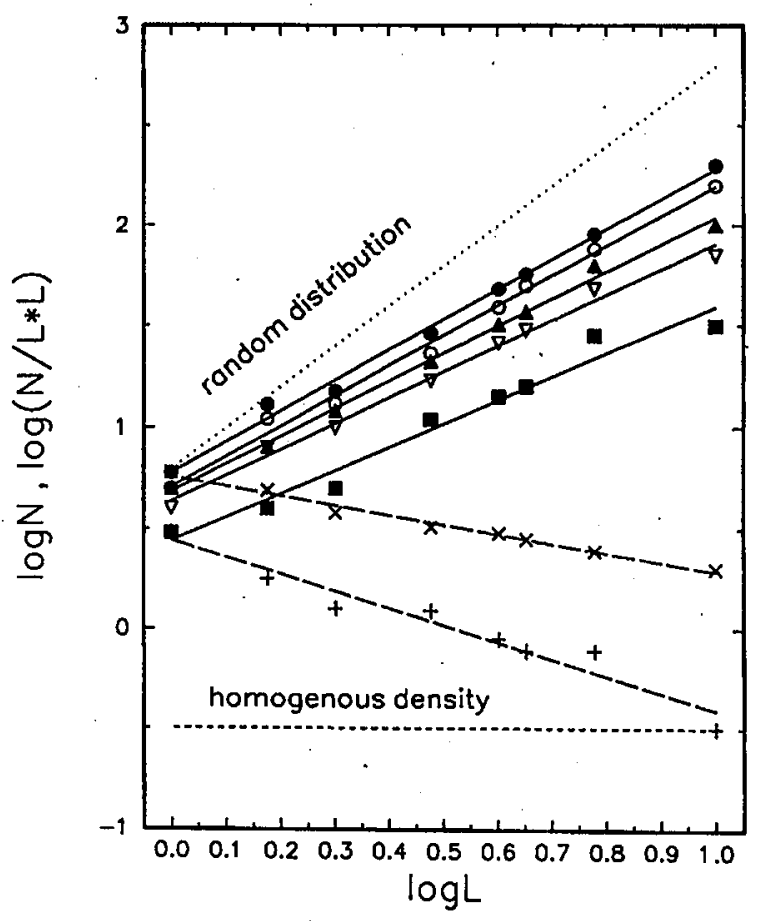

Fig. 3. The double logarithmic scale presentation of the number $N(L)$ of the defects in the square of the side $L$ (solid lines) and $N(L) /(L \times L)$ (long dash lines) for subsequent stages of evolution of defect pattern. Different symbols mark the time after the phase transition: $\bullet$ and $x-40 \mathrm{~s}, 0-50 \mathrm{~s}$, black $\triangle-70 \mathrm{~s}, \nabla-100 \mathrm{~s}$ and black $\square$ and $+-200 \mathrm{~s}$. Dotted line means $N(L)$ expected from random distribution of defects and dashed line means $N(L) /(L \times L)$ typical of homogeneous density.

The following conclusions can be formulated:

(1) The $\log N(\log L)$ plot occurs to be linear. The slope of the straight lines gives the fractal dimensionality varying from $D_{\mathrm{f}}=1.5 \pm 0.2(40 \mathrm{~s})$ to $D_{\mathrm{f}}=1.2 \pm 0.2$ 
(200 s), i.e., the values lower than $d=2$ expected for the case of homogeneous distribution of dots. The same results were obtained by presenting the $\log N /$ $(L \times L)$ vs. $\log L$ (long dash lines in Fig. 3). Thus, the distribution of defects in 2-d plane turns out to be fractally homogeneous and can be characterized by fractal dimensionality $D_{\mathrm{f}}[9]$. The $N(L)$ expected for random distribution of dots and $N(L) /(L \times L)$ for their homogeneous density are presented in Fig. 3 for comparison. The statistical self-similarity has been found for length scale changes from $0.1 \mathrm{~mm}$ to $1 \mathrm{~mm}$. For distances below $0.1 \mathrm{~mm}$ the distribution has not been checked due to difficulties in counting the points but the statistical self-similarity seems to be in force there. Due to the limitation of the sample area observed microscopically, the influence of boundary condition on the data taken at the latter stages of evolution is visible for $\log L=1$ (Fig. 3). This results in lowering the $D_{\mathrm{f}}$ value.

(2) When the analysis is stopped at $L=6 \mathrm{~cm}$ (i.e., at $\log L \approx 0.8$ ) then for all analyzed stages of evolution of nematic phase the fractal dimensionality describing the distribution of point defects turns out to be the same $\left(D_{\mathrm{f}}=1.4\right)$. Thus, the self-similar symmetry in distribution of the defects seems to be preserved rather long during the time evolution of the defect pattern, i.e., in time interval from $40 \mathrm{~s}$ till $200 \mathrm{~s}$ after the isotropic-anisotropic phase transition.

(3) In the statistical sense the general feature of the pattern of defects does not change with time. Only the characteristic length scale of the pattern increases. The growth of the areas of well-aligned molecules has been observed. This means that during evolution the nearest two defects of the opposite sign disappear first. Figure 2 illustrates the analogy between coarsening of such defect pattern and a sequence of the Sierpiński gasiket constructions in different steps of iteration arranged starting from the figure characterized by the small length scale towards the larger and larger ones. Dots in the right side of Fig. 2 represent centres of white various-sized triangles in the original Sierpiński gasket [1]. The estimated value $D_{\mathrm{f}}=1.4$ characterizing the 2 -d defects distribution is not far from the fractal dimensionality $D_{\mathrm{f}}=1.57$ [1] of the Sierpiński gasket with deterministic self-similar distribution of the various-sized white triangles. That means a rather large deviation from $d=2$ characterizing the random distribution of structural elements.

(4) The fact that distribution of defects created in the phase transition is not random means the existence of the correlation in their appearance. Due to the detection of the statistical self-similarity the degree of correlation can be estimated quantitatively.

(5) The detailed analysis of defect pattern in MBBA performed by Nagaya et al. [7] has concerned the dynamics mainly and their study of the distribution of defects has been announced to be in progress. The present paper seems to confirm their suggestion of the existence of correlation in the distribution of disclinations which they based on the experimental fact that the average value of the distances between the defects of the same sign is two times larger than between the defects of the opposite sign. When presented for the properly scaled distance the correlation function has the same form for a set of data of various images taken in different times. That corresponds to our findings that 2-d distribution of the defects is characterized by the same $D_{\mathrm{f}}$ in different moments of evolution. 


\section{Final remark}

There are earlier observations showing that evolution of 2 - $d$ string network in nematic phase [4] respects self-similarity and a surface arrangement of defects in smectic $A$ phase [10] can be characterized by $D_{\mathrm{f}}=1.5$. The usefulness of scaling hypothesis for description of coarsening of the domain wall defects was thoroughly discussed in the papers of Bray [11]. The problem seems to be vivid in cosmology where cosmic strings and other defects appearing in symmetry-breaking phase transition are used to interpret the evolution of early Universe and the origin of its large scale structure [12]. The present paper concerns the nematic phase of MBBA where the distribution of the disclinations observed in two dimensions as the ensemble of point defects is shown to be fractally homogeneous with $D_{\mathrm{f}}=1.4$.

Using the polarizing microscopy technique has the advantage that defects are observed directly. On the other hand only the situation in two dimensions can be checked. Due to complexity of the nematic medium it might be not easy to predict a three-dimensional behavior of the tangle of disclinations. To prove mathematically that geometrical arrangement of topological defects is fractal in character seems even to be a more difficult task. An explanation of the mechanism that creates a fractal-like structure and helps it to respect self-similar symmetry during evolution, is offered by a very general model of self-organized criticality proposed recently by Bak, Tang and Wiesenfeld [13]. They postulated that various large dissipative interacting systems of biology, economics, geophysics and physics when disturbed tend towards the critical state characterized by the fractal-like features. The criticality of nematic liquid crystals has ben stated by de Gennes [14]. Recently that fact has been confirmed experimentally for the nematic phase of para-azoksy-anizole (PAA) by Otnes, Riste and coworkers [15]. They analyzed the intensity of neutrons scattered on the long wavelength fluctuations of the director being influenced by distribution of disclinations in 3-d sample.

\section{Acknowledgments}

The author would like to thank Prof. Jerzy A. Janik for his encouragement, permanent interest in the work and valuable discussions.

The work is partially supported by the grant of the the Committee for Scientific Research No. $21^{\prime \prime} 03 B 04611$.

\section{References}

[1] B. Mandelbrot, Tiıe Fractal Geometry of Nature, Freeman, San Francisco 1982; M. Schroeder, Fractals, Chaos, Power Laws, Freeman and Company, New York 1991.

[2] S. Chandrasekhar, G. Ranaganath, Adv. Phys. 35, 507 (1986); N. Mermin, Rev. Mod. Phys. 51, 591 (1979); W. Bragg, Nature 133, 445 (1934).

[3] P.G. de Gennes, The Physics of Liquid Crystals, Clarendon Press, Oxford 1975, p. 132 .

[4] H. Orihira, Y. Ishibashi, J. Phys. Soc. Jpn. 55, 2151 (1986); I. Chuang, N. Turok, B. Yurke, Phys. Rev. Lett. 66, 2472 (1991). 
[5] A. Pargellis, N. Turok, B. Yurke, Phys. Rev. Lett. 67, 1570 (1991); T. Nagaya, H. Orihira, Y. Ishibashi, Phys. Soc. Jpn. 56, 3086 (1987); B. Yurke, A. Pargellis, I. Chuang, N. Turok, Physica A 178, 56 (1992); H. Toyoki, Phys. Rev. A 42, 911 (1990), I. Chuang, R. Durrer, N. Turok, B. Yurke, Science 251, 1336 (1991).

[6] Ch. Muzny, N.A. Clark, Phys. Rev. Lett. 68, 804 (1992); M.J. Bowick, L. Chander, E.A. Schiff, A.M. Srivastava, Science 263, 943 (1994).

[7] T. Nagaya, H. Hotta, H. Orihira, Y. Ishibashi, J. Phys. Soc. Jpn. 61, 3511 (1992).

[8] T. Nagaya, H. Hotta, H. Orihira, Y. Ishibashi, J. Phys. Soc. Jpn. 60, 1572 (1991).

[9] B. Mandelbrot, The Fractal Geometry of Nature, Freeman, San Francisco 1982, p. 87 .

[10] J.B. Fournier, G. Durand, J. Phys. (Paris) Colloq. 51, 157C7 (1990).

[11] A.J. Bray, in: Phase Transitions and Relaxation in Systems with Competing Energy Scales, Eds. T. Riste, D. Sherrington, Kluwer Academic Publ., Dordrecht 1993, p. 405.

[12] N. Turok, Phys. Rev. Lett. 63, 2625 (1989); C. Bauesle, Yu.M. Bunkov, S.N. Fisher, H. Godfirm, G.R. Pickett, Nature 382, 332 (1996); W.H. Zurek, Nature 317, 505 (1985); Acta Phys. Pol. B 24, 1301 (1993).

[13] P. Bak, Chao Tang, K. Wiesenfeld, Phys. Rev. A 38, 364 (1988).

[14] Ref. [3], p. 101.

[15] Z. Zhang, O.G. Mouritsen, K. Otnes, T. Riste, Phys. Rev. Lett. 70, 1834 (1993);

K. Otnes, T. Riste, Phys. Scr. T 44, 77 (1992). 